\title{
Preparation of Chitosan Material and its Antifungal Activity for Bamboo
}

\author{
Darsef $^{1}$, Suhartono $^{2}$ \\ Department of Chemistry, State University of Jakarta
}

\begin{abstract}
The main aim of present study was to prepare chitosan nanoparticle, chitosan-ZnO nanoparticle and chitosan montmorilonite to evaluate their in vitro antifungal activities. The antifungal properties of nanoparticles against phytopathogenic fungi Trametes versicolor and Tyromyces palustris were investigated at various concentrations 0.01 and $0.1 \%$. Among the various formulations of nanoparticles, chitosan-ZnO nanoparticles were found most effective at $0.1 \%$ concentration and showed 89.5 and 63.0 $\%$ growth inhibition of Trametes versicolor and Tyromyces palustris, respectively in in vitro model. At the same concentration, chitosanZnO nanoparticles also showed maximum of $87.4 \%$ inhibition rate of spore Trametes versicolor. Chitosan nanoparticles showed the maximum growth inhibitory effects $84.3 \%$ on in vitro mycelial growth of Trametes versicolor at $0.1 \%$ concentration, and $85.8 \%$ growth inhibition of Tyromyces palustris at $0.1 \%$. From our study it is evident that chitosan material particularly chitosan nanoparticle and chitosan-ZnO nanoparticles have tremendous potential for further field screening towards crop protection.
\end{abstract}

Keywords: chitosan materials, antifungal,Trametes versicolor, Tyromyces palustris

\section{Introduction}

Chitosan has emerged not only as a promising and economic source for efficient and versatile antimicrobial material but also as a biocompatible and biodegradable polymer with various applications(Geisberger et al, 2013). It has been extensively used as edible coatings to preserve the quality of many foods (Huang et al, 2012) and as a bioactive fungicide(EI Ghaouth et al, 1992). Studies have concluded that chitosan possesses antifungal activity via affinity of its cationic amino groups to cellular components.

The antifungal activity of chitosan has been reported and developed in several studies both in invitro and invivo, although chitosan activity against fungus has been shown to be less efficient as compared with its activity against bacteria (Tsai et al., 2000). The inhibitorefficiency of chitosan has been related to chitosan properties such as deacetylation degree (DD) and molecular weight (Mw). Islem et al(2014) prepared fifteen homogeneous chitosans with different acetylation degrees (DA) and molecular weights (MW). They were tested at different $\mathrm{pH}$ values for their antifungi activities against three fungi (Aspergillus niger, Fusarium oxysporum and Alternaria solani). Chitosans markedly inhibited growth of fungi tested, although the inhibitory effect depends on the type of microorganism and on the chitosan characteristics (DA and MW) with minimum inhibitory concentrations in the range of 0.001 to $0.1 \mathrm{w} \%$.For that purpose, it was examined antimicrobial activity of fifteen different chitosans perfectly soluble in acidic medium and prepared under homogeneous conditions against four Gram-negative bacteria, four Gram positive bacteria and three fungi.

Nevertheless, the bulk chitosan biopolymer has not been widely applied as antifungal agent mainly because of its insolubility in aqueous media and lower antifungal activity. To improve the antifungi activity of chitosan, researchers have prepared many derivatives which showed higher antifungi activities than chitosan. Rongchun et al (2010) describe here the preparation of three novel derivatives of chitosan including the above active groups, 2-pyridylacetyl chitosan chloride (PACS), 2-[4-(5-chloro-2hydroxybenzylideneamino)-pyridyl] acetyl chitosan chloride(CHPACS), and 2-[4-(5-bromo2hydroxybenzylideneamino-pyridylacetyl chitosan chloride (BHPACS), as well as their antifungal activity against four plant threatening pathogenic fungi Cladosporium cucumerinum, Monilinia fructicola, Colletotrichum lagenarium, and Fusarium oxysporum.. Miao et al (2014) reported that chitosan derivative coating was effective in reducing the decay of green asparagus caused by $\mathrm{F}$. concentricum. As tested in vitro, L-chitosan and H-chitosan inhibited the radial growth of F. concentricum, with a remarkable effect at a concentration of $4 \mathrm{mg} / \mathrm{ml}$, and totally inhibited spore germination at a concentration of 0.05 $\mathrm{mg} / \mathrm{ml}$, indicating that chitosan derivatives were either fungistatic or fungicidal.

Besides, the application of chitosan is limited for its poor solubility in water or high $\mathrm{pH}$ region. Therefore, taking low molecular weight chitosan with high solubility and low viscosity in water at physiologically acceptable $\mathrm{pH}$ values as starting material can enlarge the scope of application. Sudipta et al, (2014) study was to evaluate antifungal effect of water-soluble chitosan (s-chitosan) on Macrophomina phaseolina (M. phaseolina) causing jute seedling infection and monitor the change in activity of released enzymes during infection. It was observed thats chitosan enhanced the activity of defense related enzymes like chitosanase and peroxidase in the seedlings during infection by $M$. phaseolina.

Nanotechnology offers great opportunities for new antifungal additives with enhanced properties compared to conventional ones. Specific characteristics of nano-metals include high surface to volume ratio, homogeneous particles size distribution possibility of facile surface medication, good stability and the ease of preparation. These unique properties offer nano-metals great application in many fields. For example, nano-metals (e.g. silver, copper and zinc) has been added to improve fungal(Aspergillus 


\section{International Journal of Science and Research (IJSR) \\ ISSN (Online): 2319-7064}

Index Copernicus Value (2015): 78.96 | Impact Factor (2015): 6.391

brasiliensis or Penicillium funiculosum) growth resistance of building materials(Hsiao et al, 2015). Sathiyanarayanan and Muthukrishnan(2014) studied glucan nanoparticles inhibited the growth of P. aphanidermatum and suggest the use of glucan nanoparticles in prevention of diseases caused by this phytopathogenic fungus. Fabrice et al (2013) studied effect between chitosan nanogels and copper in inhibiting Fusarium graminearum growth.

Vinod et al (2015) were synthesized and evaluated $\mathrm{Cu}-$ chitosan nanoparticles for their growth promotory and antifungal efficacy in tomato (Solanum lycopersicum Mill). In pot experiments, $0.12 \%$ concentration of $\mathrm{Cu}-$-chitosan nanoparticles was found most effective in percentage efficiancy of disease control (PEDC) in tomato plants with the values of $87.7 \%$ in early blight and $61.1 \%$ in Fusarium wilt.

The objective of this work investigation, chitosan materials viz chitosan nanoparticle, and chitosan- $\mathrm{ZnO}$ nanoparticle and chitosan-montmorilonite which were further examined against phytopathogenic fungi viz.

\section{Materials and Methods}

\subsection{Chemicals}

Sodium tripolyphosphate, sodium hydroxide, was supllied by Sigma Co and used as received. Glacial acetic acid and hydrochloric acid were of analytical grade, Chitosan DD 90 was purchased from Material Science Research Centre, BATAN, Jakarta and montmorilonite from Laboratory Petrologi, Trisakti University chemistry laboratory, State University of Jakarta).

Fungal strains Trametes versicolor and Tyromyces palustris Were obtained from Laboratory of Micro- biology,State University of Jakarta,Indonesia and used for antimicrobialassays.

\subsection{Preparation Chitosan Nanoparticle(ChNp)}

$\mathrm{ChNp}$ was prepared by the ionic gelation process based on the report by Calvo et al (1997), with severalmodifications. The chitosan solutions with mass concentrations $2.5 \mathrm{~g} / \mathrm{L}$ were prepared by dissolving purified chitosanin $1 \%(\mathrm{w} / \mathrm{w})$ acetic acid $2 \%$ solution with sonication at $800 \mathrm{~W}$ for 15 $\min$. The $\mathrm{pH}$ of chitosan solutions was adjusted to 4.5 with $\mathrm{NaOH}$ 1.0 M solution. The chitosan NP suspension was spontaneously formed by adding dropwise the $50 \mathrm{~mL}$ sodium tripolyphosphate $1.5 \mathrm{~g} / \mathrm{L}$ solution into the $150 \mathrm{~mL}$ chitosan solution under a magnetic stir of $250 \mathrm{rpm}$ at room temperature for 60 min.centrifuged at $10,000 \times$ gfor $30 \mathrm{~min}$ at room temperature in order to separate the nanoparticles from large particles or aggregates or freeze dried to obtain powder samples.. After centrifugation sediment and supernatantwere separated by carefully removing the supernatant layer.

\subsection{Preparation chitosan/ZnO nanoparticle}

The appropriate amount of $1.0 \mathrm{~g} \mathrm{ZnO}$ powder was dissolved in $100 \mathrm{~mL}$ of $1 \%(\mathrm{v} / \mathrm{v})$ acetic acid and changed to zinc cations. Then $1.0 \mathrm{~g}$ of chitosan was added to the above solution. The mixture was sonicated for $30 \mathrm{~min}$ after magnetic stirring and then the acidity adjusted by $0.1 \mathrm{M}$ $\mathrm{NaOH}$ solution $(\mathrm{pH} 4.5)$. The clear sol was obtained after keeping overnight at room temperature. Afterwards, the resulting sol was added to $0.1 \mathrm{M} \mathrm{NaOH}$ solution,stirrer for $12 \mathrm{hr}$ at room temperature. Then the color became light white. Chitosan/ $\mathrm{ZnO}$ nanoparticles were obtained through sufficiently washing with deionized water and dried in an infrared oven.

\subsection{Preparation of chitosan-montmorilonite (Chi-MMT)}

Chitosan solution was prepared by dissolving $4 \mathrm{~g}$ of chitosan in $196 \mathrm{~mL}$ of $2 \% \mathrm{v} / \mathrm{v}$ acetic acid. The $\mathrm{pH}$ of chitosan solution was adjusted to 4.9 with $1 \mathrm{M} \mathrm{NaOH}$. The solution was left overnightbefore was slowly added into a suspension bath containing $100 \mathrm{ml}$ montmorilonite $2.50 \%(\mathrm{w} / \mathrm{v})$. The mixture was treated at $60^{\circ} \mathrm{C}$ for $24 \mathrm{hr}$. The chitosan intercalated MMT (Chi-MMT)was washed with distilled water until the $\mathrm{pH}$ of the washed water became neutral. ChiMMT was separated from water by centrifugation at 3500 $\mathrm{rpm}$ for $10 \mathrm{~min}$ and then dried at $60^{\circ} \mathrm{C}$ for $48 \mathrm{hr}$.

\subsection{Antifungal activities}

Different concentrations ( 0.01 and $0.1 \%$, w/v) of various nanoparticles in aqueous solution were used in antifungal activity test against twofungus species Trametes versicolor (L: Fr.), Fomitopsis palustris.Potato dextrose agar medium was prepared and poured in Petri dishes $(90 \mathrm{~mm} \times 15 \mathrm{~mm})$, with above mentioned percentages of various nanoparticles, separately. Mycelial bit fromperipheral end of uniform size (diameter, $5.0 \mathrm{~mm}$ ) was taken from7 days old culture of test pathogens and placed in the centre of test Petri dishes. All the Petri dishes were incubated at $28 \pm 1{ }^{\circ} \mathrm{C}$ for 7 days and the observation of radial mycelial growth was recorded when controlled Petri dish cover full growth $(90 \mathrm{~mm})$. All the treat-ments consisted of three replications and experiment was repeated twice. The inoculated plates were compared with control (without nanoparticles) to calculate the \% inhibition rate of mycelia of the pathogen by using the formula given by Vincent.

$$
\% \text { Inhibition rate }=\frac{\mathrm{Mc}-\mathrm{Mt}}{\mathrm{Mc}} \times 100 \%
$$

where $\mathrm{Mc}$ is the mycelial growth in control, $\mathrm{Mt}$ is the mycelial growth in treatment

\subsection{Spore germination method}

The antifungal activities of nanoparticles (0.001, 0.005, $0.01,0.02,0.06$ and $0.1 \%, \mathrm{w} / \mathrm{v})$ on spore germination of Trametes versicolor were tested. Spore suspension $\left(1.0 \times 10^{3}\right.$ spores $/ \mathrm{ml}$ ) of Trametes versicolor prepared aseptically from 7 days old pure culture; $50 \mu$ l of spore suspension and 50 $\mu l$ of nanoparticle at above mentioned concentrations in aqueous were taken on glass slides in 10 replicates. All the treatments were maintainedat $28 \pm 1{ }^{\circ} \mathrm{C}$ for $8 \mathrm{~h}$ and the observations

were made under microscope to calculate the \% inhibition rate by counting the number of spore germinated compared to control. 


\section{International Journal of Science and Research (IJSR)}

ISSN (Online): 2319-7064

Index Copernicus Value (2015): 78.96 | Impact Factor (2015): 6.391

$$
\% \text { Inhibition rate }=\frac{\mathrm{Gc}-\mathrm{Gt}}{\mathrm{Gc}} \times 100 \%
$$

where Gc is the germination in control and Gt is the germination intreatment.

\subsection{Decay test procedure for bamboo}

The treated bamboo specimens, beech (Fagus orientalis L.) was tested for decay resistance according to Indonesian National standard (SNI). Brown-rot fungi, Trametes versicolor (L: Fr.), and white-rot fungi, Fomitopsis palustris, were grown in $250 \mathrm{ml}$ glass bottles to establish an active mycelium. The treated and untreated bamboo were sterilised in an autoclave for $20 \mathrm{~min}$, and then bamboo blocks were placed in bottles containing actively growing cultures of either Fomitopsis palustris or Trametes versicolor. Five repetitions were collected for each test group. The bottles were incubated for 12 weeks at $25^{\circ} \mathrm{C}$ and $70 \%$ relative humidity.

After incubation, the test blocks were withdrawn from the culture bottles. The mycelium was carefully brushed off of the blocks. The bamboo blocks were then dried for $24 \mathrm{~h}$ at room temperature and stored at $105^{\circ} \mathrm{C}$ overnight. After oven-drying, the final weight of each bamboo block was acquired. The weight loss due to decay was calculated as a percentage determined using the dry weight loss of each bamboo block before and after incubation.

\section{Results and Discussion}

\subsection{FTIR analysis of ChNp}

Fig. 1 shows the FTIR spectra of ChNP, in the wave number range of $4000-600 \mathrm{~cm}^{-1}$. For pure chitosan. the characteristic bands due to the stretching vibration of $-\mathrm{NH} 2$ and $-\mathrm{OH}$ groups were observed at $3356 \mathrm{~cm}^{-1}$. The feature peak at $1547 \mathrm{~cm}^{-1}$ for amide II (N-H bending vibration) and the small shoulder peak at $1647 \mathrm{~cm}^{-1}$ for amide I (-CO stretching vibration) indicate the high degree of deacetylation of chitosan. The flattening of the amine peak at $3356 \mathrm{~cm}^{-1}$ in Fig1. indicates that majority of amino group of chitosan participated in the electrostatic interaction with TPP(data not show).

\subsection{Characterization of chitosan/ $\mathrm{ZnO}$ nanoparticle}

FTIR analysis was performed to confirm the interaction of chitosan, ZnO-chitosan.Bare chitosan was characterizedwith some specific peaks located at 1643, 903 and $3308,8 \mathrm{~cm}^{-}$ ${ }^{1}$ which related to amide $\left(\mathrm{CONH}_{2}\right)$, anhydro glucosidic ring and primary amine $\left(\mathrm{NH}_{2}\right)$, respectively.

The main characteristic peaks of $\mathrm{CS}-\mathrm{ZnO}$ at $3333,14 \mathrm{~cm}^{-1}$ signify the stretching modes of $\mathrm{NH}_{2}$ and $\mathrm{OH}$ group. The peaks at 2978 and $2885 \mathrm{~cm}^{-1}$ denote the stretching modes of asymmetric stretching of $\left(\mathrm{CH}_{3}\right)$ and $\left(\mathrm{CH}_{2}\right)$ in the CS network while those at 1625.10 and $1317.44 \mathrm{~cm}^{-1}$ were attributed to CQO stretching vibration and scissoring vibration of $-\mathrm{NH} 2$. In addition, a broad absorption peak at the range of 580-400 $\mathrm{cm}^{-1}$ was ascribed to the vibration of the $\mathrm{O}-\mathrm{Zn}-\mathrm{O}$ group. While the spectrum of chitosan(Fig.3) showed peaks at $3000-3750 \mathrm{~cm}^{-1}$ due to the overlapping of $\mathrm{O}-\mathrm{H}$ and $\mathrm{N}-\mathrm{H}$ stretching bands, $2920 \mathrm{~cm}^{-1}$ for aliphatic $\mathrm{C}-\mathrm{H}$ stretching, 1634 and $1594 \mathrm{~cm}^{-1}$ for $\mathrm{N}-\mathrm{H}$ bending, 1420 and $1382 \mathrm{~cm}^{-1}$ for $\mathrm{C}=\mathrm{O}$ bending, 1151 and $1079 \mathrm{~cm}^{-1}$ for $\mathrm{C}-\mathrm{O}$ stretching.

\subsection{FTIR analysis of ChMmt}

The spectrum of the Chi-MMT shows the combination of characteristic absorptions due to the chitosan and MMT groups. The peak at $1594 \mathrm{~cm}^{-1}$ of the $-\mathrm{NH}_{2}$ group in the starting chitosan was shifted to $1520 \mathrm{~cm}^{-1}$ in the Chi-MMT spectrum, corresponding to the deformation vibration of the protonated amine group $\left(-\mathrm{NH}_{3}{ }^{+}\right)$of chitosan. This $\left(-\mathrm{NH}_{3}{ }^{+}\right)$ group interactswith the negatively charged sites of MMT.

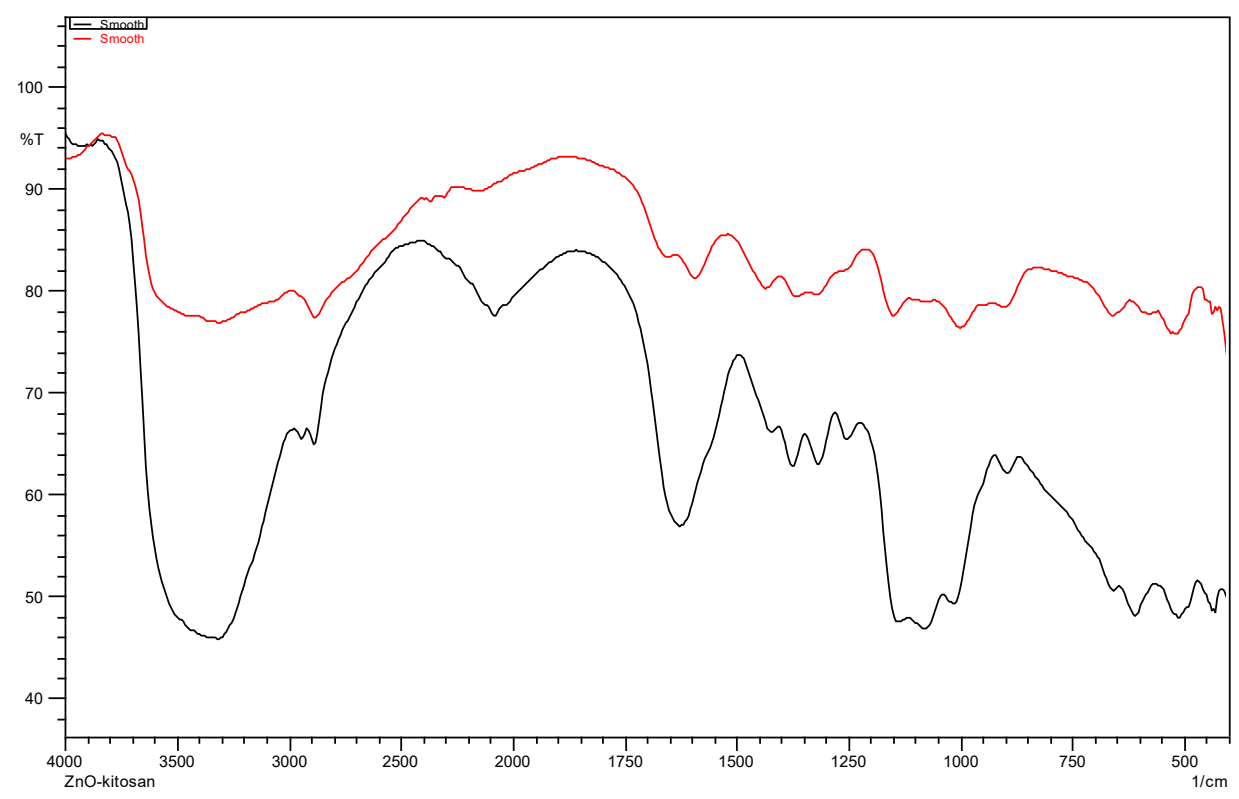

Volume 6 Issue 1, January 2017 www.ijsr.net 


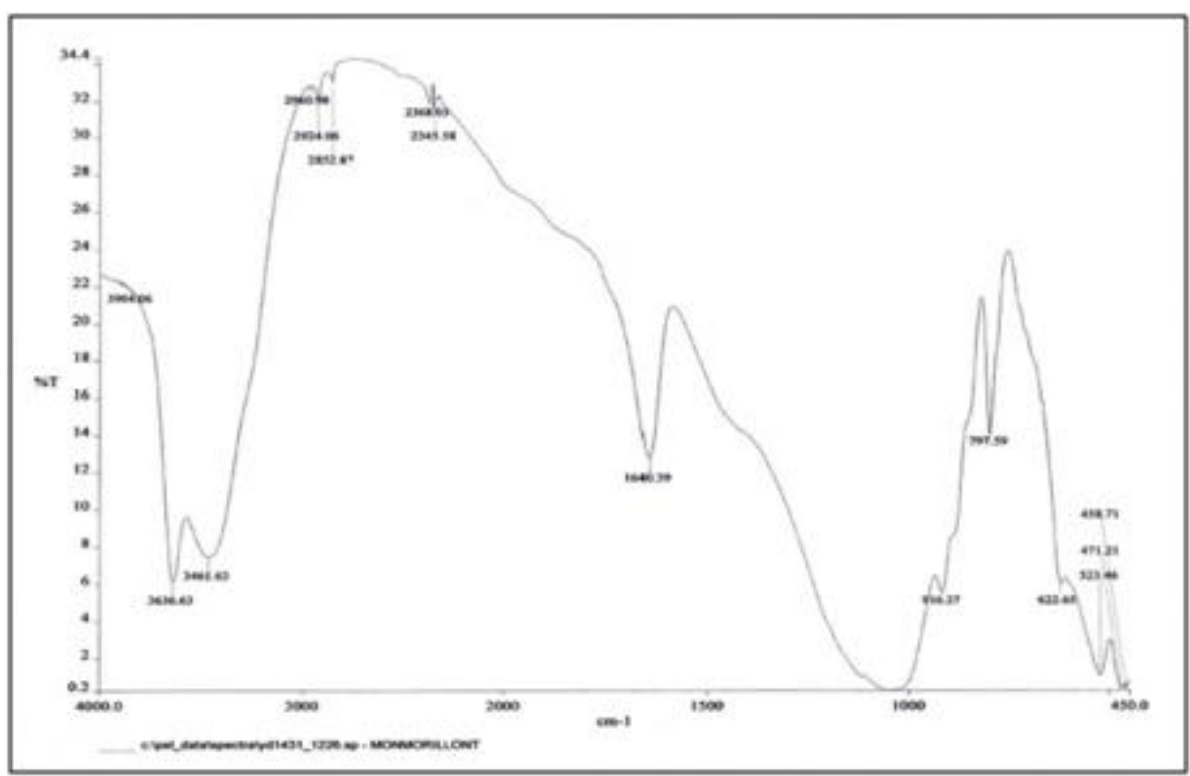

Figure 1: FTIR of (a) chitosan and (b) chitosan/ZnO nanoparticle

Invitro mycelial growth of Trametes versicolor was comprehensively controlled by 0.01 and $0.1 \%$ concentrations of all the chitosan. A maximum $89.5 \%$ inhibition rate was recorded at $0.1 \%$ concentration of chitosan- $\mathrm{ZnO}$ nanoparticle followed by $84.3 \%$ at $0.1 \%$ chitosan nanoparticle. In the case of chitosan montmorilonite, $81.6 \%$ of mycelial growth was inhibited at $0.1 \%$ concentration

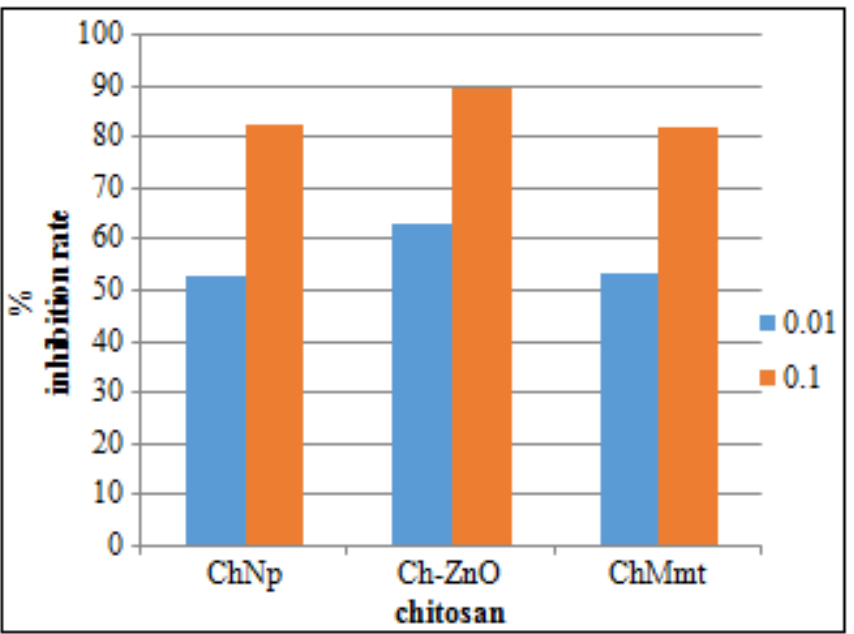

Figure 4: Effect of various chitosan and concentration on in vitro mycelial growth of Trametes versicolor

Chitosan nanoparticle displayed strong inhibition of mycelial growth of Fomitopsis palustris. A maximum 85.8\% inhibition rate was recorded at $0.1 \%$ of chitosan nanoparticle. However, 0.01 and $0.1 \%$ concentrations showed similar growth inhibition rate as per the statistical analysis. Chitosan montmorilonite,showed a dose dependent effect on mycelial growth. The highest inhibition rate $(64.4 \%)$ was recorded at $0.1 \%$ concentration while the other concentrations 0.01 gave inhibition rate from 36.8. Chitosan $\mathrm{ZnO}$ nanoparticle at $0.01 \%$ concentrations inhibited growth from $53.0 \%$ whereas $0.1 \%$ formulation inhibited $63.0 \%$ of mycelia growth
A maximum $87.4 \%$ spore germination was inhibited by $0.1 \%$ concentration of chitosan- $\mathrm{ZnO}$ nanoparticles followed by chitosan $(86.4 \%)$ at $0.1 \%$. Taken as a whole, all the examined nanoparticles were found effective in controlling spore germination ofTrametes versicolor. Bulk chitosan nanoparticle, chitosan- $\mathrm{ZnO}$ nanoparticle at $0.1 \%$ level were found less effective for inhibition of mycelial growth and spore germination

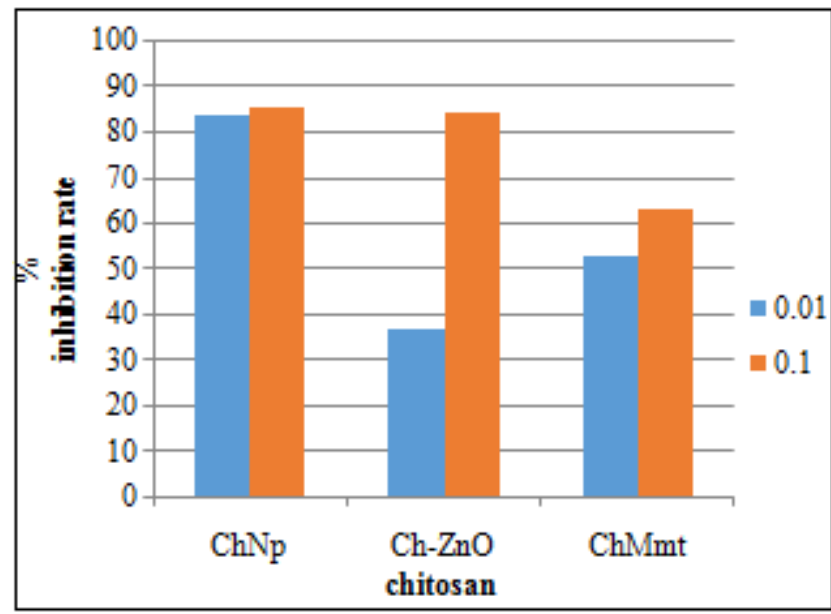

Figure 5: Effect of various chitosan and concntration on in vitro mycelial growth of Fomitopsis palustris

The mechanism, which chitosan affects the growth and survival of fungi, may be related to its ability to interfere with the negatively charged residues of macromolecules exposed on fungal surfaces forming polyelectrolytic complexes, thereby affecting membrane permeability and causing leakage of intracellular electrolytes and proteinaceous constituents. Additionally, it has been proposed that the inhibitory efficacy of chitosan against pathogenic fungi in bamboo could also be related to its ability to increase the production of defense-related enzymes (e.g., chitinase and $\beta$-1,3-glucanase) and antioxidant activity (e.g., polyphenol oxidase and peroxidase) in chitosan coated bamboo. 


\section{International Journal of Science and Research (IJSR) \\ ISSN (Online): 2319-7064 \\ Index Copernicus Value (2015): 78.96 | Impact Factor (2015): 6.391}

\subsection{Weight loss of bamboo}

The weight loss of un-aged (control) beech specimens was found to $39.5 \%$ for Trametes versicolor, and 32.1 for Fomitopsis palustris attack, respectively. These weight loss results verified that the decay test was valid according to SNI. Weight loss caused by Trametes versicolor attack was found to be $10.2 \%$ and 6.9 for aged and control specimens with node sections, and 5.4 and $7.9 \%$ for aged and control specimens without node sections, respectively. Weight loss after Fomitopsis palustris attack of aged specimens with nodes, control specimens with nodes, aged specimens without nodes, and control specimens without nodes were in the ranges $3.4-10.7 \%$, respectively. Expectancy of service life of bamboo specimens varied from 2 to $3 \mathrm{yr}$ to $8 \mathrm{yr}$, while the expectancy of service life of wood specimens was service life against Trametes versicolor and Fomitopsis palustris.

\section{Conclusions}

Chitosan nanoparticles, chitosan-ZnO nanoparticles and Chitosan montmorilonite were prepared and their structures were well characterized by FTIR.. The antifungal capacity of chitosan is real but complicated to control. The effectiveness of chitosan does not only depend on the chitosan formulation but also on the fungus type. The antifungal activity of three chitosan materials were effective

Chitosan $\mathrm{ZnO}$ nanoparticles contribute to their higher antifungal activity against Trametes versicolor in in vitro studies.Chitosan nanoparticles also showed maximum inhibition rate of spore germination of Trametes versicolor. The effiancy of chitosan based nanoparticles mainly chitosan and $\mathrm{ZnO}$-chitosan could further be tested on other plant pathogenic fungi in in vitro and in vivo models. The present studyis a effort to explore potential of chitosan based nanoparticles andthe findings of present study could be utilized for development ofnano fungicide for crop protection in future.

\section{Acknowledgements}

The authors would like to thank Ministry of Education of Government of Indonesia for its financial support with research grant hibah bersaing 2014 .

\section{References}

[1] EI Ghaouth, A, Arul, J., Grenier, J., \& Asselin, A. (1992). Antifungal activity of chitosan on two posthaverst pathogens of strawberry fruits. Phytopathology, 82, 398-402

[2] Fabrice Brunel, Nour Eddine El Gueddari, Bruno M. Moerschbacher(). Complexation of copper(II) with chitosan nanogels: Toward antifungal efficacy of nanometals supported $\mathrm{TiO} 2$ and ozone on the resistant Aspergillus niger spore Journal of Hazardous Materials 261, 155-162

[3] Geisberger, G., Gyenge, E. B., Hinger, D., Kach, A., Maake, C., \& Patzke, G. R. (2013). Chitosan- thioglycolic acid as a versatile antimicrobial agent. Biomacromolecules, 14, 1010-1017

[4] Huang, J. Y., Chen, Q. C., Qiu, M., \& Li, S. Q. (2012). Chitosan-based edible coatings for quality preservation of postharvest whiteleg shrimp (Litopenaeus vannamei). Journal of Food Science, 77, C491-C496

[5] Hsiao-Lin Huang, Chi-Chi Lin, Kunnan Hsu(2015). Comparison of resistance improvement to fungal growth on green and conventional building materials by nanometal impregnation .Building and Environment 93, 119-127

[6] Islem Younes, Sabrine Sellimi , Marguerite Rinaudo , Kemel Jellouli , Moncef Nasri (2014) . Influence of acetylation degree and molecular weight of homogeneous chitosans on antibacterial and antifungal activities particles and its antifungal activity International Journal of Biological Macromolecules 70, $440-443$

[7] Kuo et al (2013) assessed the antifungal effectiveness of these nanometals supported $\mathrm{TiO} 2$ catalysts on $\mathrm{A}$. niger spores. A. niger was selected because it is ubiquitous in the air in indoor environments.

[8] Kuo-Pin Yu*, Yi-Ting Huang, Shang-Chun Yang. (2013) .The Complexation of copper(II) with chitosan nanogels: Toward control of microbial growth Carbohydrate Polymers 92, 1348-1356

[9] Miao Qiu, Chu Wu, Gerui Ren, Xinle Liang, Xiangyang Wang $\Uparrow$, Jianying Huang(2014).Effect of chitosan and its derivatives as antifungal and preservative agents on postharvest green asparagus. Food Chemistry 155, 105111

[10] Rongchun Li, Zhanyong Guo, Pingan Jiang (2010). Synthesis, characterization, and antifungal activity of novel quaternary chitosan derivatives . Carbohydrate Research 345, 1896-1900

[11] Sudipta Chatterjeea,b,*, Bishnu P. Chatterjeec, Arun K. Guha(2014). A study on antifungal activity of watersoluble chitosan against Macrophomina phaseolina. International Journal of Biological Macromolecules 67, 452-457

[12] Sathiyanarayanan Anusuya, Muthukrishnan Sathiyabama. Preparation of -d-glucan nan International Journal of Food Microbiology 185 57-63

[13] Tsai, G.J., Su, W.H., Chen, H.C., Pan, C.L., 2002. Antimicrobial activity of shrimp chitin and chitosan from different treatments and applications of fish preservation. Fish. Sci. 68, 170-177.

[14] Vinod Saharana, Garima Sharmaa, Meena Yadava, Manju Kumari Choudharya, S.S. Sharma , Ajay Pal, Ramesh Raliya, Pratim Bisw.(2015) .Synthesis and in vitro antifungal efficacy of $\mathrm{Cu}$-chitosan nanoparticles against pathogenic fungi of tomato International Journal of Biological Macromolecules 75 , 346-35 